\title{
THE CRYSTAL STRUCTURE OF BIS[BENZYLTRIPHENYLPHOSPHONIUM]HEXACHLOROTIN(IV) DICHLOROFORM SOLVATE
}

\author{
Dainis Dakternieks ${ }^{*},{ }^{1}$ Bernhard Zobel, ${ }^{1}$ and Edward R. T. Tiekink ${ }^{\star 2}$
}

${ }^{1}$ Centre for Chiral and Molecular Technologies, Deakin University, Geelong, Victoria, Australia 3217

¿ Department of Chemistry, The University of Adelaide, Australia 5005

<dainis@deakin.edu.au> <edward.tiekink@adelaide.edu.au>

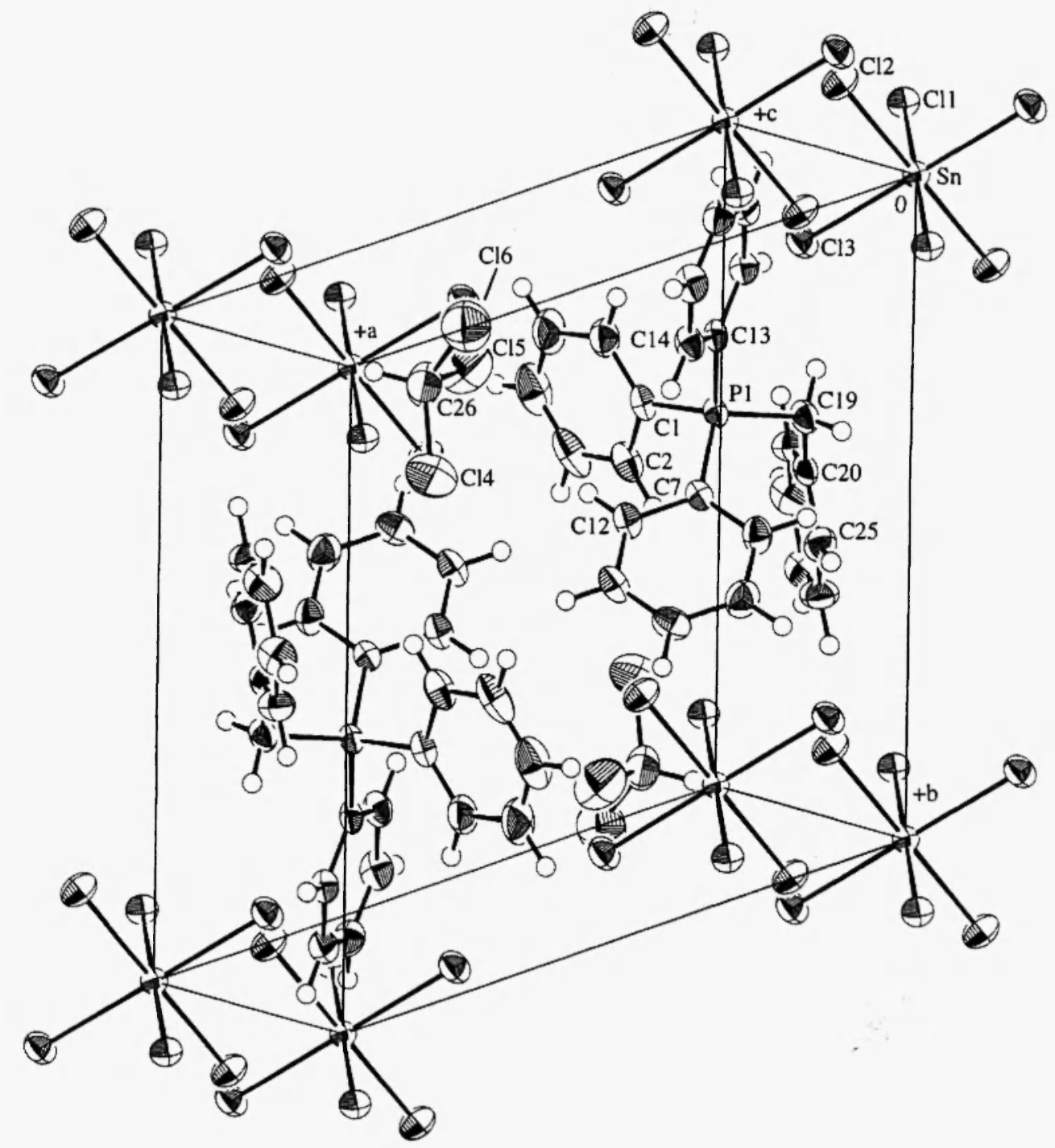

Figure 1. Unit cell contents and atomic labeling scheme for $\left[\left(\mathrm{PhCH}_{2}\right) \mathrm{Ph}_{3} \mathrm{P}\right]_{2}\left[\mathrm{SnCl}_{6}\right] .2 \mathrm{CHCl}_{3}(50 \%$ probability ellipsoids). Selected bond distances and angles: $\mathrm{Sn}-\mathrm{Cl}(1) 2.436(1), \mathrm{Sn}-\mathrm{Cl}(2) 2.424(1)$, $\mathrm{Sn}-\mathrm{Cl}(3)$ 2.441(1) $\dot{A} ; \mathrm{Cl}(1)-\mathrm{Sn}-\mathrm{Cl}(2)$ 90.72(4), Cl(1)-Sn-Cl(2)i 89.28(4), Cl(1)-Sn-Cl(3) 89.29(4), $\mathrm{Cl}(1)-$ $\mathrm{Sn}-\mathrm{Cl}(3)^{i} 90.71(4), \mathrm{Cl}(2)-\mathrm{Sn}-\mathrm{Cl}(3)$ 89.28(5), $\mathrm{Cl}(2)-\mathrm{Sn}-\mathrm{Cl}(3)^{i}$ 90.72(5) 


\section{Comment}

The compound is a rearrangement product isolated from a solution containing $\left[\left(\mathrm{PhCH}_{2}\right) \mathrm{Ph}_{3} \mathrm{P}_{2}\left[\mathrm{Cl}_{4} \mathrm{Sn}\left(\mathrm{CH}_{2}\right)_{8} \mathrm{SnCl}_{4}\right]\right.$ [1]. The anion is centrosymmetric and is almost a regular octahedron. The most significant intermolecular contact in the lattice occurs between the $\mathrm{Cl}(2)$ and the chloroform-H atoms so that $\mathrm{Cl}(2) \ldots \mathrm{H}^{\prime \prime}$ is $2.72 \AA, \mathrm{Cl}(2) \ldots \mathrm{C}(26)^{\prime \prime}$ is $3.565(5) \bar{A}$ and the $\mathrm{Cl}(2) \ldots \mathrm{H}^{\prime \prime}$. $C(26)^{\prime \prime}$ angle is $149^{\circ}$; symmetry operation ii: $1-x,-y, 1-z$.

\section{Experimental}

Preparation: Colourless crystals (m.p. $266-267^{\circ} \mathrm{C}$ ) were grown at room temperature from a $\mathrm{CH}_{2} \mathrm{Cl}_{2} / \mathrm{CHCl}_{3}(1: 5)$ solution of $\left[\left(\mathrm{PhCH}_{2}\right) \mathrm{Ph}_{3} \mathrm{P}_{2}\left[\mathrm{Cl}_{4} \mathrm{Sn}\left(\mathrm{CH}_{2}\right)_{8} \mathrm{SnCl}{ }_{4}\right]\right.$ prepared from 2 mole equivalents of [ $\left.\left(\mathrm{PhCH}_{2}\right) \mathrm{Ph}_{3} \mathrm{P}\right] \mathrm{Cl}$ and $\mathrm{Cl}_{3} \mathrm{Sn}\left(\mathrm{CH}_{2}\right)_{8} \mathrm{SnCl}_{3}$. A sample to be used for elemental analysis was kept at $60^{\circ} \mathrm{C}$ and $10^{-3}$ Torr for $1 \mathrm{~h}$ to remove all volatiles. Anal: Calcd for $\mathrm{C}_{50} \mathrm{H}_{44} \mathrm{Cl}_{2} \mathrm{P}$ Sn, $\mathrm{C}, 57.86 ; \mathrm{H}, 4.27$. Found: C 57.82, H 4.39\%. 'H NMR (299.98 MHz, CD $\mathrm{OD}) \delta 4.94\left(\mathrm{~d}, 2 \mathrm{H},{ }^{2} \mathrm{~J}\left({ }^{31} \mathrm{P} \cdot{ }^{1} \mathrm{H}\right)=15 \mathrm{~Hz}, \mathrm{CH}_{2}\right)$; 6.98-7.94 (mm, 20H, Ph). ${ }^{13} \mathrm{C}\left\{{ }^{1} \mathrm{H}\right\}$ NMR $\left(75.44 \mathrm{MHz}, \mathrm{CD}_{3} \mathrm{OD}\right) \delta 30.54\left(\mathrm{~d}, 1 \mathrm{C},{ }^{1} \mathrm{~J}\left({ }^{31} \mathrm{P}-{ }^{13} \mathrm{C}\right)=48 \mathrm{~Hz}\right)$; $118.80\left(\mathrm{~d}, 3 \mathrm{C}, \mathrm{J}\left({ }^{31} \mathrm{P}-{ }^{13} \mathrm{C}\right)=86 \mathrm{~Hz}\right) ; 128.51\left(\mathrm{~d}, 1 \mathrm{C}, \mathrm{CJ}^{2}\left({ }^{3} \mathrm{P}-{ }^{13} \mathrm{C}\right)=8 \mathrm{~Hz}\right) ; 129.57$ (d, $1 \mathrm{C}, 5 \mathrm{~J}\left({ }^{31} \mathrm{P}-\right.$ $\left.\left.{ }^{13} \mathrm{C}\right)=4 \mathrm{~Hz}\right) ; 129.82\left(\mathrm{~d}, 2 \mathrm{C},{ }^{\mathrm{n} J}\left({ }^{31} \mathrm{P}-{ }^{13} \mathrm{C}\right)=3 \mathrm{~Hz}\right) ; 131.16\left(\mathrm{~d}, 6 \mathrm{C},{ }^{n} \mathrm{~J}\left({ }^{31} \mathrm{P}-{ }^{13} \mathrm{C}\right)=12 \mathrm{~Hz}\right) ; 132.09(\mathrm{~d}$, $\left.2 \mathrm{C},{ }^{n} \mathrm{~J}\left({ }^{31} \mathrm{P}-{ }^{13} \mathrm{C}\right)=5 \mathrm{~Hz}\right) ; 135.18\left(\mathrm{~d}, 6 \mathrm{C},{ }^{n} \mathrm{~J}\left({ }^{31} \mathrm{P}_{-}{ }^{3} \mathrm{C}\right)=10 \mathrm{~Hz}\right) ; 136.18\left(\mathrm{~d}, 3 \mathrm{C},{ }^{4} \mathrm{~J}\left({ }^{31} \mathrm{P}-{ }^{33} \mathrm{C}\right)=3 \mathrm{~Hz}\right)$.

${ }^{119} \mathrm{Sn}\left\{{ }^{1} \mathrm{H}\right\}$ NMR (111.85 $\left.\mathrm{MHz}, \mathrm{CD}_{3} \mathrm{OD}\right) \delta$-652.3.

\section{Crystallography:}

Table 1. Crystal data for $\left[\left(\mathrm{PhCH}_{2}\right) \mathrm{Ph}_{3} \mathrm{P}\right]_{2}\left[\mathrm{SnCl}_{6}\right] \cdot 2 \mathrm{CHCl}_{3}$

\begin{tabular}{|c|c|c|c|}
\hline $\begin{array}{l}\text { Formula } \\
\text { Crystal system } \\
\text { Space group } \\
b, A \\
\alpha,{ }^{\circ} \\
\gamma,{ }^{\circ} \\
\text { Temperature, } \mathrm{K} \\
\text { Trans. factors } \\
F(000) \\
\text { Reflns meas. } \\
\text { Reflns with } I>3.0 \sigma(I) \\
R(F), R_{\mathrm{w}}(\mathcal{F}) \\
\text { Programs used } \\
\text { Deposition number }\end{array}$ & $\begin{array}{l}\mathrm{C}_{52} \mathrm{H}_{46} \mathrm{Cl}_{12} \mathrm{P}_{2} \mathrm{Sn} \\
\text { triclinic } \\
\text { Pbar1 } \\
12.027(3) \\
92.01(2) \\
73.33(2) \\
1 \\
173 \\
0.928-1 \\
642 \\
6687 \\
4099 \\
0.039,0.044 \\
\text { teXan [2], DIFABS [3], DI } \\
\text { CCDC 141374 }\end{array}$ & 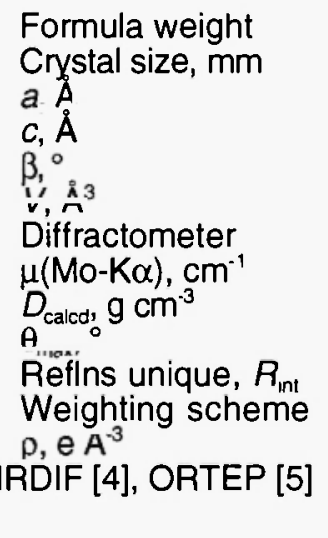 & $\begin{array}{l}1277.0 \\
0.07 \times 0.10 \times 0.18 \\
11.757(3) \\
10.307(2) \\
96.09(2) \\
1388.2(6) \\
\text { AFC7R } \\
11.29 \\
1.527 \\
27.5 \\
6380,0.034 \\
1 /\left[\sigma^{2}\left(F_{0}\right)+0.00017 F^{2} .\right. \\
0.69\end{array}$ \\
\hline
\end{tabular}

\section{Acknowledgments}

Support from the Australian Research Council is gratefully acknowledged.

\section{References}

[1] B. Zobel, A. Duthie, D. Dakternieks, K. Jurkschat and E.R.T. Tiekink Organometallics submitted for publication.

[2] teXsan, Single Crystal Structure Analysis Software, Version 1.04 (1997), Molecular Structure Corporation, The Woodlands, TX, USA.

[3] N. Walker and D. Stuart, Acta Crystallogr., A39 (1983) 158.

[4] P.T. Beurskens, G. Admiraal, G. Beurskens, W.P. Bosman, S.R.O. Garcia-Granda, R.O. Gould, J.M.M. Smits and C. Smykalla, The DIRDIF program system, Technical Report of the Crystallography Laboratory, University of Nijmegen, The Netherlands, 1992.

[5] C.K. Johnson, ORTEP. Report ORNL-5138 (1976), Oak Ridge National Laboratory, TN, USA.

Received: March 3, 2000 - Accepted: March 13, 2000 Accepted in revised camera-ready format: March 14, 2000 